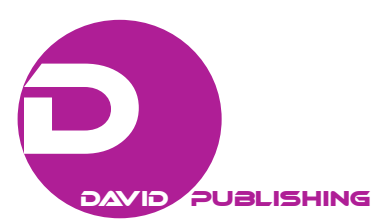

\title{
Development of Distributed Complex Network for Representation of Knowledge Base
}

\author{
Archil Prangishvili, Roman Samkharadze, Medea Tervdoradze \\ Lia Gachechiladze, Nino Lolashvili \\ Georgian Technical University, Tbilisi, Georgia
}

\begin{abstract}
For management of power system on the bases of distributed complex network (DCN-network) authors developed a new structure of knowledge base: at macro-nodes of network are located a database and a logical conclusion making machine, and on the arcs are located their connecting properties. Such structure excludes transitions from a logical conclusion making machine to database. It provides opportunity of fast and efficient processing of information, modeling of complex processes etc.
\end{abstract}

Keywords: distributed macro-network, production rules, power system mode, knowledge base

\section{Introduction}

One of the complicated, multi-criteria systems is power system, management, development, and modernization of which requires a new method. Increasing of the accuracy of power systems models, complication of network schemes, tightening of requirements to quality, and economy of the electrical energy and other factors, require usage of new expert's knowledge-based management systems in the energetics.

For a lot of subject areas, which require description by an analytical and simulation models, priority and conflict situations are essential and determinant. In particular, such events occur during management of power system modes. For solving of such type of tasks traditional networks capacity is not enough. The article proposes a distributed complex network for solving tasks of power systems modes’ effective management.

\section{The Main Content}

While developing expert systems semantic network is used for presentation of knowledge in graphic view. By the means of the semantic network we can create a knowledge base. From the basic features of the semantic networks we can distinguish: the opportunities, describing the relations between objects, which are given in the network' nodes; the possibility of using semantic networks for creating of structures and objects, as well as rules of knowledge base. Thus, semantic networks, as usually, have a describing character.

There are a lot of problematic areas, description of which by the semantic or other types of networks is not

\footnotetext{
Archil Prangishvili, doctor of Science, academician, Georgian Technical University, Tbilisi, Georgia.

Roman Samkharadze, doctor of Science, professor, Georgian Technical University, Tbilisi, Georgia.

Medea Tervdoradze, Ph.D., professor, Georgian Technical University, Tbilisi, Georgia.

Lia Gachechiladze, Ph.D., assistant professor, Georgian Technical University, Tbilisi, Georgia.

Nino Lolashvili, Ph.D., associative professor, Georgian Technical University, Tbilisi, Georgia.

Correspondence concerning this article should be addressed to Roman Samkharadze, Department of Computer Engineering, Georgian Technical University, 77 Kostava Street, Tbilisi 0175, Georgia.
} 
informative, and it is necessary to bring in new types of networks. For example, during management of power system's modes, capacity of semantic networks is not enough for describing of the decision-making processes, because it is impossible to solve conflict situations, to describe organization of priority requirements' service etc.

To overcome the mentioned difficulties it offered a new kind of network - the distributed complex network (DSN-Network), by means of which we can determine a new structure of knowledge base: at macro-nodes of network are located the database and the logical conclusion making machine, and on the arcs are located the connecting properties. Such structure excludes transitions from a logical conclusion making machine to database. To macro-nodes are given logical functions of network's development. The macro-nodes are presented as priority-conflict networks. In macro-nodes are placed markers. Network development, i.e. the direction of marker motion, depends on its own parameters and network’s dynamic parameters.

\section{Definition.}

DSN-network is the seven,

$$
\mathrm{DSN}=<\mathrm{ND}, \mathrm{AR}, \mathrm{MN}, \mathrm{RP}, \mathrm{SP}, \mathrm{EF}, \mathrm{MM}>
$$

where ND—multitude of network's nodes, AR—multitude of arcs of network, MN—set of network macro-nodes, RP-route prehistory, SP-productions system, EF-multitude of Evaluation functions, $\mathrm{MM}$ — set of management modes.

The multitude of nodes ND consists of sub-multitudes of nodes ND' and ND". Nodes ND' coincide with notion of nodes of traditional semantic networks, nodes are marked by circles and have names:

$$
\mathrm{ND}^{\prime}=\left\{\mathrm{r}_{1}^{\prime}, \mathrm{r}_{2}^{\prime}, \ldots, \mathrm{r}_{\mathrm{m}}^{\prime}\right\}
$$

For the subject areas (we mean energy objects) the node can be: "Dispatcher (D)" (which, provides a dialogue between an expert system and a power system's dispatcher, Figure 1);

Besides of nodes of traditional semantic networks it is imported concept of "macro-nodes" (ND"):

$$
\mathrm{ND}^{\prime \prime}=\left\{\mathrm{r}_{1}, \mathrm{r}_{2}, \ldots, \mathrm{r}_{\mathrm{m}}{ }^{\prime \prime}\right\}
$$

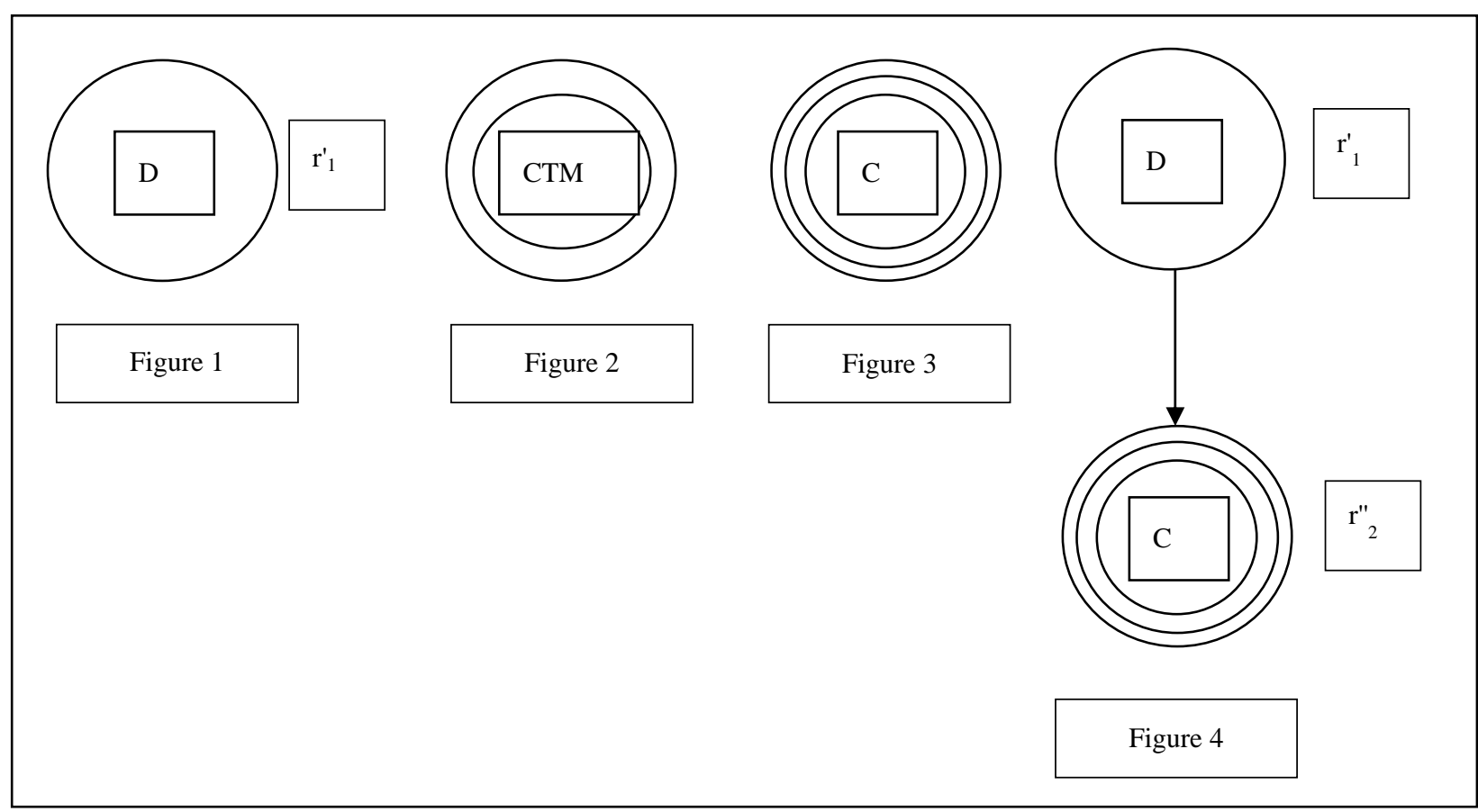


The necessity of determination of macro-nodes is conditioned by a new architectural and structural capabilities of the network, reflecting the possibility of combining in one macro-node of the logical conclusion making machine and the database. Such organization gives the possibility of decentralization of operations in the network on the level of architectural possibilities for physical structures with type "multiprocess or systems with cellular-automatic architecture". The combination of the new network principles and the architectural capabilities allow reducing response time in such networks, which is very important while managing energy objects. The examples of macro-nodes: "Comparison of two modes (CTM)" (Selection of the best daily modes) (Figure 2), "Calculations (C)” (Generation of daily modeby initial data) (Figure 3), etc.

Introducing the concept of a network arc coincides with the traditional notion of semantic networks arc. Arc' examples for energy objects may be: “select”, “dialogue”, “execute”, etc. (Figure 4).

The set of macro-nodes is the new concept of DSN-network. It implies the repetition of macro-nodes, which has the same names and the same functions, but a variety of input information, for example, "computing”, "evaluation of mode”, etc. These macro-nodes may be used in different tracts of macro-network.

Prehistory of the routes lies in the fact that there is remembering route, which will take the place of marker. The essence of prehistory of the route lies in the fact that it is possible to remember the route, which passes the marker (point, moving in the network). The route includes all positions of the marker before getting to the given position. Introduction of prehistory of route gives possibility to stop calculations in a node, which we need, and to remember the previous calculation results. For example, after generating the daily mode, the previous results can be accessed without repeated calculations, and therefore without wasting of computer resources, as well as continue execution in DSN-network from necessary point. The concept of prehistory gives us the opportunity of being trusted and/or necessary moving from one node to another taking into account the semantics of sub-routing.

The productions system includes a standard elements of procedures, in particular - the global database, a multitude of production rules and management system. Given system of productions opens its essence in macro-nodes, macro-nodes contain all elements of the productions system. Production rules' multitude contains IF-THEN production rules.

Also it is introduced the concept of evaluation function. They are assigned to nodes of macro-net. The role of the evaluation functions is in reduction of search volume in a large size network. This is achieved by introducing of such quality criteria, which allows us to move in the best direction. For example, if generated daily mode is not accepted by the appropriate quality criteria, then a new daily mode will be generated.

Mode of management allows us to choose the best mode by decision making in the case of a variety of data, depending on the length of the network. It contains the following types of search, like: search in the depth, width, from top to bottom, from bottom to top, etc.

Using such structure of the knowledge base followings can be performed:

- Effective information processing (by time);

- Modeling of complex processes;

- Separating such subsets of the network, which are particularly important in the learning' mode, (have not only a descriptive property, but also are related to the decision-making procedures — set of macro nodes with their connections), which is recommended, for example, during creating simulators for power systems' dispatchers. 
The distributed complex network (macro-network) developed for management of the daily modes of power systems contains the following macro-nodes: "Entering and checking of initial data", "Changing and checking of initial data", "Determining of factors", "Determining of situations”, "Calculations", "Estimating of modes", "Comparing of two modes", "Loading of thermal power plant to $\mathrm{N}_{\min }$ power", "Liquidation of the negative imbalances", "Loading of thermal power plant to $\mathrm{N}_{\max }$ power". Macro-node may include macro-network or PCN-network. The macro-node "Calculations" includes the macro-network, which contains three macro-nodes: "Loading of thermal power plant to $\mathrm{N}_{\min }$ power", "Liquidation of the negative imbalances", "Loadingof thermal power plant to $\mathrm{N}_{\max }$ power". All these three macro-nodes and above noted macro-nodes contain PCN-networks (priority conflict-networks) (Prangishvili \& Samkharadze, 2002).

The macro-node "Entering and checking of initial data" implements entering and checking of data validity. If all data are entered correctly, then management will be transferred to "Dispatcher" macro-node. Dispatcher selects one of the three alternatives: either re-enters the initial data, or changes them, or starts calculations. The macro-node "Changing and checking of initial data" implements changing and checking of data validity. After change of the data management will be transferred to the macro-node "Dispatcher". The dispatcher selects one of three alternatives: either re-enters data, or changes them, or starts calculations.

The macro-node "Determining of factors" determines four main factors, which describe existing situations.

The macro-node "Determining of situation" performs a determination of one situation from the possible 16. During this determination will select the appropriate model, vector criterion, and the priorities for local criteria.

Then, we move to the macro-node "Calculations" for performing of calculations. This macro-node contains the macro-network, which consists of three macro-nodes. First time we will performe the macro-node "Load of thermal power plant to $\mathrm{N}_{\text {maxwanted }}$ power". As a result, the thermal power plant (TPP) at each hour generates $\mathrm{N}_{\text {maxwanted }}$ power. Then, system performes macro-node "Liquidation of the negative imbalances".

As a result, it will perform the daily load of hydroelectric power station in all or possible hours. If negative imbalances have been liquidated, then we leave macro-node "Calculations". Otherwise, we will performe the macro-node "Load of thermal powerplant to $\mathrm{N}_{\max }$ power". Then, again system performes macro-node "Liquidation of the negative imbalances" and exits from macro-node "Calculations". Such kind of branches performs the macro-node "Branching function 1". In result of calculations the first mode is generated. It performs its evaluation by priority criteria and management transfers to macro-node "Dispetcher". Dispatcher selects one of three alternatives: choose the result, enter, or change initial data.

If there were generated second, third, etc. modes, then after their evaluation the last result will be chosen, or it will be compared with the best previous result. Such kind of branches performs the macro-node "Branching function 2". This macro-node chooses the one from two branches. If the first mode is generated, then it will be evaluated and the management will be transferred to macronode "Dispatcher". If it generated the second, third, etc. modes, then after evaluation of these modes its comparison will be performed with the best previous mode and choose the better one.

The macro-node "Comparing of two modes" performs comparison of two modes and chooses the better one. Then, management transfers to the macro-node "Dispatcher" and dispatcher chooses one of three possible alternatives: choose the result generated by expert system, enter initial data, or change them.

Knowledge base consists of seven sets of production rules (Samkharadze, Gasitashvili, Prangishvili, \& Verulava, 2000). These rules are nonconflict and nonexcess. They are: the initial data checking, the factors 
determining, the situation determining, defining of model and choicing of a vector criterion, determination of the structure of advantages, solving of the vector task of mathematical programming, estimating of the mode, comparison of the modes and choice of the better one. Such structure of knowledge significantly accelerates the search process of the desired rule and therefore increases the efficiency of the work of the expert system.

Expert knowledge is the main source of knowledge about multi-criterial system for the expert system. For representation of knowledge is used production model. The rules are of the form: "IF...THEN". The part IF of the rule contains one or more conditions. The part THEN of the rule contains action, may consist of one or more conclusions. The IF and THEN parts of a rule can contain AND/OR boolean functions. The part IF of the rule contains one or more conditions, which may be associated with each other using AND/OR functions. AND function notes with $\wedge$ character, OR function with $\vee$.

Each rule is the independent fragment of knowledge about object, in which all conditions of using of the rule are obviously defined in the part IF.

As mentioned above, the knowledge base consists of production rules and facts. Facts can be constant and dynamic. Which fact will be dynamic during a day depends on the initial information. Knowledge base consists of the following facts:

- In peak hours consumption of electricity is high,

- Spring is characterized by abundance of water in rivers,

- Winter is characterized by sparse water in rivers,

- Night-time electricity consumption is low,

- In winter electricity consumption is high,

- In summer electricity consumption is less,

$\cdots$

- The ability of regulation of thermal stations is lower.

Clarification of the rules and elimination of obstacles between them is implemented during working of expert system.

The fragment of set of production rules of checking initial data is as following:

IF maximum power of hydropowerplant (HPP) is less than the minimum,

THEN correction of HPP's maximum and minimum power will be performed;

IF HPP's hourly generation is greater than the maximum power,

THEN correction of hourly generation will be performed;

IF hydropower station's maximum power is zero,

THEN hydropower station is disabled during a day;

$\cdots$

IF hourly number of thermal power station's working aggregates is greater than maximum or less than the minimum,

THEN correction of hourly number of aggregates will be performed.

The fragment of set of production rules for determining of main factors is as follows:

IF $\Psi<0$, THEN mode is deficient;

$\cdots$

IF $Q>24 \times Q_{t}^{\max }$, THEN HPP has abundance of water. 
Here, $\Psi$ is system's imbalance, $Q$ is planned water consumption for given HPP for given day, $Q_{t}^{\max }$ is maximum water consumption for given HPP in thour.

The fragment of production rules for determination of the situation is as follows:

IF $\operatorname{Ex}\left(\overline{u_{1}}\right) \wedge \operatorname{Ex}\left(\overline{u_{2}}\right) \wedge \operatorname{Ex}\left(\overline{u_{3}}\right) \wedge \operatorname{Ex}\left(\overline{u_{4}}\right)$, THEN $\operatorname{Ex}\left(s_{1}\right) ;$

$\cdots$

IF $\operatorname{Ex}\left(\mathrm{u}_{1}\right) \wedge \operatorname{Ex}\left(\mathrm{u}_{2}\right) \wedge \operatorname{Ex}\left(\mathrm{u}_{3}\right) \wedge \operatorname{Ex}\left(\mathrm{u}_{4}\right)$, THEN $\operatorname{Ex}\left(\mathrm{s}_{16}\right)$.

Here, $\mathrm{Ex}$ - the predicate of existence, factor $\mathrm{u}_{1}$ means, that electricity consumption is high, $\overline{\mathrm{u}_{1}}$ —electricity consumption is not great, $\mathrm{u}_{2}$ - on hydropower stations is much water, $\overline{\mathrm{u}_{2}}$ - on hydropower stations is little water, $\mathrm{u}_{3}$ - on TPP is much fuel, $\overline{\mathrm{u}_{3}}$ - on TPP is a little fuel, $\mathrm{u}_{4}$-from the water complex there is a demand of water, $\overline{\mathrm{u}_{4}}$-from the water complex there is not a demand of water. The Ex $\left(\mathrm{u}_{1}\right)$ means existence of factor $\mathrm{u}_{1}$.

The production rule for determination of the structure of advantages is as follows:

IF $\operatorname{Ex}\left(F_{11}^{1}(\mathrm{X})\right)$, THEN $\operatorname{Pr}\left(\mathrm{f}_{1}(\mathrm{X}), \mathrm{f}_{2}(\mathrm{X})\right)$.

Here, $\mathrm{Pr}$-predicate, which determines priorities, $F_{11}^{1}(\mathrm{X})$ - vector criteria, $\mathrm{f}_{1}(\mathrm{X})$ - the local criteria for minimizing of the energy imbalance, $\mathrm{f}_{2}(\mathrm{X})$ - the local criteria for maximisation of generation of HPP.

The production rule of choice of model and vector criteria is as follows:

IF $\operatorname{Ex}\left(s^{1}\right)$, THEN Ch $\left(z_{1}^{1}, F_{11}^{1}(\mathrm{X})\right)$.

Here $s^{1}$-the first situation, $\mathrm{Ch}-$ the predicate of choice, $\mathrm{Ch}\left(z_{1}^{1}, F_{11}^{1}(\mathrm{X})\right)$ means, that it will select the model $z_{1}^{1}$ and the vector criteria $F_{11}^{1}(\mathrm{X})$.

The fragment of production rules' set for solving of mathematical programming's vector task is as follows:

IF Ex( $\left.s^{1}\right)$, THEN Wo(1);

$\cdots$

IF $\operatorname{Ex}\left(\mathrm{s}^{16}\right)$, THEN Wo(16).

The Wo(1) means, that works the first algorithm;

IF HPP is lack of water,

THEN HPP at first time covers the peak part of load graph, then as far as possible covers the remainder part of the load graph;

IF peak hour,

THEN all HPP will generate the maximum power;

IF HPP has abundance of water,

THEN (HPP will generate the maximum power in all hours of a day) $\wedge$ (HPP does not take part in regulation of load);

IF there is a demand on water from the water complex,

THEN (Not satisfied with the demand on water) $\vee$ (Content with the demand on water);

$\cdots$

IF content with the demand of water,

THEN HPP minimizes water consumption.

\section{Conclusions}

In the article for management of power system's modes, complex distributed network is proposed. Each macro-node can contain macro-network or PCN-network. The knowledge base consists of seven sets of 
production rules: initial datachecking, factors determining, the situation determining, defining of model and choice of a vector criterion, determination of the structure of advantages, solving of the vector task of mathematical programming, estimating of the mode, comparison of modes and choice of the better one. Such structure of knowledge significantly accelerates the search process of the desired rule and therefore increases the efficiency of the work of the expert system.

\section{References}

Prangishvili, A., \& Samkharadze, R. (2002). The theory of constructing an expert systems for power systems management. Monograph. Tbilisi. Sciences, p. 285.

Samkharadze, R., Gasitashvili, Z., Prangishvili, A., \& Verulava, I. (2000). About constructing the distributed complex network. GeorgianTechnical University. Proceedings. \#3 (431). Tbilisi. 